\title{
The value of radiography in the diagnosis of closed damage the spleen
}

\begin{abstract}
Observed 106 patients with closed damage the spleen. A certain value in the timely recognition of the nature of the catastrophe in the abdominal cavity had a panoramic radiography of the abdomen. When the injury of the spleen is constantly observed the high standing the left dome of the diaphragm. In doubtful observations performed laparoscopy.
\end{abstract}

Keywords: trauma, spleen, sightseeing, radiography, iris
Volume 5 Issue I - 2018

\author{
Shaposhnikov VI \\ Department of Oncology, Russia
}

Correspondence: Shaposhnikov VI, Department of Oncology with a course of radiation Diagnostics and therapy the NIGHT, Kuban Medical Institute, Krasnodar, Russia, Email 79183446404@yandex.ru

Received: December 27, 2017 | Published: February 14, 2018

\section{Introduction}

There are isolated, combined and multiple mechanical damage to the spleen. They are closed and open., ${ }^{1,2}$ If an isolated only traumatized spleen, when multiple - Other authorities in the same system, when combined - the tissues and organs of other systems. In closed injuries the integrity of the skin and mucous membranes has been saved. These damages may be complete (at the same time, damaged parenchyma, and the capsule) and incomplete, when torn or only the capsule, or the parenchyma of the body with the formation of The subcapsular hematomas, which in the future may or organize, or break free in the abdominal cavity, that is happens, the so-called two-minute gap. ${ }^{3}$ Distinguish The breakdown spleen, break it from the vascular legs, damage to one of its poles and open the gate of the Authority. Isolated closed damage the spleen is accompanied by symptoms of traumatic shock and acute blood loss, and in the future, and PERITONITIS. When combined same injuries, of which the most often there are fractures of the bones of osteo-articular system and traumatic brain injury, the gravity of the status of the affected increases dramatically. It should be noted that sometimes the symptoms that accompany the combined damage, outwardly manifested more manifestly compared with symptoms on the part of the traumatized spleen, which can lead to serious medical error, both in terms of early diagnosis and the formulation of rational treatment algorithm. In this process a negative role plays and the concomitant alcoholic intoxication. ${ }^{1-4}$ In the medical literature the issues involved, in our opinion, the neglected. This has led us to undertake a special study.

\section{Material and methods}

In this work, presented the results of the treatment of 106 patients with closed injuries spleen, operated in Private hospitals of Krasnodar region between the ages of 17 and 72years of age, of which men was $80(75.4 \%)$ patients with an isolated injury to the spleen was $92(86.6 \%)$, and with the combined-14(13.4\%), of which the fracture of the ribs on the left was at 6 , the sternum is at 2 , the left shoulder is at 2 and 4 severe traumatic brain injury .

\section{Results and discussion}

Of the total number of victims (106) died of $4(3.8 \%)$. If an isolated injury mortality rate was $2.1 \%$ (of 92 patients died of (2), and when combined - $14.2 \%$ (from 14 patients died 2 from traumatic brain injury). If an isolated injury to the earliest symptom of damage to the spleen was unconscious condition and only then the symptom "Grandpa - Stand Up - ca" and the pose on the left side with squeezed to the stomach legs. On palpation noted the soreness in the left half of the stomach and the rigidity of the abdominal wall, and the percussion as defined in the fatigue was sloping places the abdomen. Gradually nastupala pallor of the skin. If the blood loss is rapidly evolved, anemia, hypotension and tachycardia, The shock quickly index reached 1.0 and above. In the review of radiography of the abdominal and thoracic cavities and indicated the backlog the left dome of the diaphragm and its high standing compared with the right bubble, fade and blasting contours of the bowel. When пальцевом study of colorectal cancer was defined overhanging the front of her walls, and the women in the rear puncture of the vagina was blood. The diagnostic tests allowed $90(84.9 \%)$ of the affected to recognize the injury of the spleen during the first hour after hospitalization. The 16(15.1\%) patients, and they all had the trauma of coinfection, damage to the spleen is detected only after 4-7hours of receipt in the hospital. At this time, they were treated or from damage to the osteoarticular apparatus, or traumatic brain injury. However, the condition they have deteriorated. The affected suspected closed trauma of the abdomen. This would require the review of radiography of the abdomen. It identified the characteristic symptoms of damage to the spleen. In 2 patients with an isolated injury to the belated diagnosis of due to the heavy alcoholic drunkenness. Providers unnecessarily waiting for their sober up. X-ray examination was done too late. Both of the patient died of hemorrhagic shock. Splenetomy was carried out in $101(95.2 \%)$ of the patient, and SUTURE the capsule body with wrapping up his seal scalp - in $5(4.8 \%)$. The average duration of treatment was 11.3 bed days.

\section{Conclusion}

Closed combined damage to the spleen are characterized by difficulties in the recognition that is associated with the dominance of the influence of symptoms other traumatized organs and tissues over the symptoms on the part of this body. For this reason, the significantly (up to 6-7hours) late diagnosis of such damage, and the mortality rate increases to 6.7 times compared to an isolated injury. The crucial importance of the timely recognition of the nature of the 
catastrophe in the abdominal cavity has a thorough examination of the victim, while the review of radiography of the abdominal cavity need special attention paid to the height of the standing the left dome of the diaphragm, and the questionable observations to perform laparoscopy.

\section{Acknowledgements}

None.

\section{Conflict of interest}

Author declares that there is no conflict of interest.

\section{References}

1. Aleksey Ermolov AS. Abdominal trauma Guide to emergency surgery of the abdominal cavity. The clinic and diagnostics. 2006. p. 425-433.

2. Lebedev NV, Abakumov MM, Malyarchuk VN. Diagnosis of stomach damage when combined injury. Surgery. 2002;12:53-58.

3. Sokolov VA. Multiple somatic and injury. Medicine 2006. 512p.

4. Tereshchenko OA, Tarakanov VA, Poleyev AV, et al. Organosaving methods for abdominal trauma with damage to parenchymatous organs Complications in surgery of diseases and abdominal injuries. Materials of the scientific-practical conference, plenum of the board ROEH-SPb. EFA medica. 2014. p. 400-402. 